Y. HIRASAWA

KODAI MATH. J.

6 (1983), 70-79

\title{
ON SOLUTIONS OF A HOMOGENEOUS LINEAR MATRIX EQUATION WITH VARIABLE COMPONENTS
}

\author{
By YOSHIKAZU HiRASAwa
}

1. We denote the totality of real numbers by $\mathbf{R}$ and the totality of complex numbers by $\mathbf{C}$.

Let $I$ be a closed interval $[\alpha, \beta]=\{t \mid \alpha \leqq t \leqq \beta, t \in \mathbf{R}\}$. We denote by $C^{\mu}(I, \mathbf{R})$ the totality of real-valued functions defined and of class $\mathrm{C}^{\mu}$ on $I(\mu=0,1, \cdots, \infty)$, and hereafter we fix some $\mu$.

A complex-valued function $f(t)$ defined on $I$ is called a function of class $\mathrm{C}^{\mu}$ on $I$ if $\operatorname{Re} f(t) \in C^{\mu}(I, \mathbf{R})$ and $\operatorname{Im} f(t) \in C^{\mu}(I, \mathbf{R})$. We denote by $C^{\prime \prime}(I, \mathbf{C})$ the totality of complex-valued functions defined and of class $\mathrm{C}^{\mu}$ on $I$.

A $d$-dimensional row vector $\boldsymbol{x}$ with components $x_{\rho} \in \mathbf{C}(\rho=1,2, \cdots, d)$ will be denoted by

$$
\boldsymbol{x}=\left(x_{1}, x_{2}, \cdots, x_{d}\right)
$$

and a $d^{\prime}$-dimensional column vector $\boldsymbol{y}$ with components $y_{\sigma} \in \mathbf{C}\left(\sigma=1,2, \cdots, d^{\prime}\right)$ by

$$
\boldsymbol{y}=\left(\begin{array}{c}
y_{1} \\
y_{2} \\
\vdots \\
y_{d^{\prime}}
\end{array}\right)=\operatorname{col}\left(y_{1}, y_{2}, \cdots, y_{d^{\prime}}\right)
$$

Now, let $B(t)$ be a square matrix of degree $n$ :

$$
B(t)=\left(\begin{array}{cccc}
b_{11}(t) & b_{12}(t) & \cdots & b_{1 n}(t) \\
b_{21}(t) & b_{22}(t) & \cdots & b_{2 n}(t) \\
\vdots & \vdots & & \vdots \\
b_{n 1}(t) & b_{n 2}(t) & \cdots & b_{n n}(t)
\end{array}\right),
$$

where $b_{j k}(t) \in C^{\mu}(I, \mathbf{C})(\jmath, k=1,2, \cdots, n)$, and let us assume, throughout this paper, that for a positive integer $s: 1 \leqq s \leqq n-1$, a condition

$$
\operatorname{rank} B(t)=n-s(=r)
$$

is satisfied on the interval $I$, and further let us consider a homogeneous linear matrix equation

$$
B(t) P(t)=O
$$

where $P(t)$ is an $n \times s$ matrix:

Received March 1, 1982 


$$
P(t)=\left(\begin{array}{cccc}
p_{11}(t) & p_{12}(t) & \cdots & p_{1 s}(t) \\
p_{21}(t) & p_{22}(t) & \cdots & p_{2 s}(t) \\
\vdots & \vdots & & \vdots \\
p_{n 1}(t) & p_{n 2}(t) & \cdots & p_{n s}(t)
\end{array}\right) .
$$

The purpose of this paper is to establish the existerice of solutions $P(t)$ of the equation (2) on $I$, such that every component $p_{\jmath k}(t)$ of $P(t)$ belongs to $C^{\mu}(I, \mathbf{C})$ and

on $I$.

$$
\operatorname{rank} P(t)=s
$$

Y. Sibuya treated such a problem in a paper [1], provided that $B(t)$ was periodic on $-\infty<t<+\infty$. But as a certain part of the proof was omitted in his paper, we will, in this paper, give a detailed proof of this part to clarify the matter.

2. Let $I_{1}$ and $I_{2}$ be two open intervals $\left(\alpha_{1}, \beta_{1}\right)$ and $\left(\alpha_{2}, \beta_{2}\right)$ contained in the interval $I$, such that $\alpha_{1}<\alpha_{2}<\beta_{1}<\beta_{2}$, and but let us consider $I_{1}=\left[\alpha_{1}, \beta_{1}\right)$ if $\alpha_{1}=\alpha$ and $I_{2}=\left(\alpha_{2}, \beta_{2}\right]$ if $\beta_{2}=\beta$. that

Put $r=n-s$ and let $B\left(\begin{array}{llll}j_{1} & j_{2} & \cdots & j_{r} \\ k_{1} & k_{2} & \cdots & k_{r}\end{array}\right)$ denote a minor of degree $r$ of $B(t)$ such

$$
\begin{aligned}
& B\left(\begin{array}{cccc}
j_{1} & j_{2} & \cdots & j_{r} \\
k_{1} & k_{2} & \cdots & k_{r}
\end{array}\right)=\left|\begin{array}{cccc}
b_{\jmath_{1} k_{1}}(t) & b_{\jmath_{1} k_{2}}(t) & \cdots & b_{\jmath_{1} k_{r}}(t) \\
b_{\jmath_{2} k_{1}}(t) & b_{\jmath_{2} k_{2}}(t) & \cdots & b_{\jmath_{2} k_{r}}(t) \\
\vdots & \vdots & & \vdots \\
b_{\jmath_{r} k_{1}}(t) & b_{\jmath_{r} k_{2}}(t) & \cdots & b_{\jmath_{r} k_{r}}(t)
\end{array}\right|, \\
& \left(\begin{array}{c}
1 \leqq j_{1}<j_{2}<\cdots<j_{r} \leqq n \\
1 \leqq k_{1}<k_{2}<\cdots<k_{r} \leqq n
\end{array}\right) .
\end{aligned}
$$

Let us now assume that the condition (1) is satisfied on $I_{1} \cup I_{2}$ and further that a condition

$$
B\left(\begin{array}{cccc}
j_{1} & j_{2} & \cdots & \jmath_{r} \\
k_{1} & k_{2} & \cdots & k_{r}
\end{array}\right) \neq 0
$$

is satisfied on $I_{1}$ and a condition

$$
B\left(\begin{array}{cccc}
l_{1} & l_{2} & \cdots & l_{r} \\
m_{1} & m_{2} & \cdots & m_{r}
\end{array}\right) \neq 0
$$

is satisfied on $I_{2}$.

Under these assumptions, it is clear that there exist two $n \times s$ matrices $P(t)$ and $Q(t)$ having the following properties:

(I) $P(t)$ and $Q(t)$ satisfy matrix equations

and

$$
B(t) P(t)=O \quad \text { on } \quad I_{1}
$$




$$
B(t) Q(t)=O \quad \text { on } \quad I_{2}
$$

respectively, and every component $p_{j k}(t)$ of $P(t)$ belongs to $C^{\mu}\left(I_{1}, \mathbf{C}\right)$ and every component $q_{j k}(t)$ of $Q(t)$ belongs to $C^{\mu}\left(I_{2}, \mathbf{C}\right)$;

(II) $\operatorname{rank} P(t)=s$ on $I_{1}$ and $\operatorname{rank} Q(t)=s$ on $I_{2}$.

However, we here wish to look over the structure of these matrices $P(t)$ and $Q(t)$.

Let us define $s$-tuple $\left(j_{r+1}^{\prime}, j_{r+2}^{\prime}, \cdots, j_{n}^{\prime}\right)$ for $1 \leqq j_{1}<j_{2}<\cdots<j_{r} \leqq n$ in such a manner that $1 \leqq j_{r+1}^{\prime}<j_{r+2}^{\prime}<\cdots<j_{n}^{\prime} \leqq n$ and $\left\{\jmath_{1}, \cdots, \jmath_{r}, j_{r+1}^{\prime}, \cdots, j_{n}^{\prime}\right\}=\{1,2, \cdots, n\}$. That is, $\jmath_{1}<\jmath_{2}<\cdots<j_{r}$ and $j_{r+1}^{\prime}<j_{r+2}^{\prime}<\cdots<j_{n}^{\prime}$ form a complete system of indices $\{1,2, \cdots, n\} . \quad s$-tuples $\left(k_{r+1}^{\prime}, k_{r+2}^{\prime}, \cdots, k_{n}^{\prime}\right), \quad\left(l_{r+1}^{\prime}, l_{r+2}^{\prime}, \cdots, l_{n}^{\prime}\right)$ and $\left(m_{r+1}^{\prime}, m_{r+2}^{\prime}, \cdots, m_{n}^{\prime}\right)$ are defined for $1 \leqq k_{1}<k_{2}<\cdots<k_{r} \leqq n, 1 \leqq l_{1}<l_{2}<\cdots<l_{r} \leqq n$ and $1 \leqq m_{1}<m_{2}<\cdots<m_{r} \leqq n$ in the same manner.

By virtue of Cramer's rule, we see the following fact.

We can take arbitrarily all components $p_{k_{\sigma}^{\prime} g}(t)$ of $s$ row vectors

$$
\hat{\boldsymbol{p}}_{k_{\sigma}^{\prime}}(t)=\left(p_{k_{\sigma}^{\prime} 1}(t), p_{k_{\sigma}^{\prime} 2}(t), \cdots, p_{k_{\sigma}^{\prime} s}(t)\right) \quad(\sigma=r+1, r+2, \cdots, n),
$$

or $s$ column vectors

$$
\tilde{\boldsymbol{p}}_{\boldsymbol{g}}(t)=\operatorname{col}\left(p_{k_{r+1}^{\prime} g}(t), p_{k_{r+2}^{\prime}}(t), \cdots, p_{k_{n}^{\prime} g}(t)\right) \quad(g=1,2, \cdots, s)
$$

under restrictions that $p_{k_{\sigma}^{\prime} g}(t) \in C^{\mu}\left(I_{1}, \mathbf{C}\right)$ and

$$
\operatorname{det}\left(\tilde{\boldsymbol{p}}_{1}(t), \tilde{\boldsymbol{p}}_{2}(t), \cdots, \tilde{\boldsymbol{p}}_{s}(t)\right)=\operatorname{det}\left(\begin{array}{c}
\hat{\boldsymbol{p}}_{k_{r+1}^{\prime}}(t) \\
\hat{\boldsymbol{p}}_{k_{r+2}^{\prime}}(t) \\
\vdots \\
\hat{\boldsymbol{p}}_{k_{n}^{\prime}}(t)
\end{array}\right) \neq 0 \quad \text { on } \quad I_{1} .
$$

We can further express other $r$ row vectors

$$
\hat{\boldsymbol{p}}_{k_{\rho}}(t)=\left(p_{k_{\rho^{1}}}(t), p_{k_{\rho^{2}}}(t), \cdots, p_{k_{\rho} s}(t)\right) \quad(\rho=1,2, \cdots, r)
$$

by linear combinations of $\hat{\boldsymbol{p}}_{k_{\sigma}^{\prime}}(t)(\sigma=r+1, r+2, \cdots, n)$ :

$$
\hat{\boldsymbol{p}}_{k_{\rho}}(t)=\sum_{\sigma=r+1}^{n} \xi_{\rho \sigma}(t) \hat{\boldsymbol{p}}_{k_{\sigma}^{\prime}}(t) \quad(\rho=1,2, \cdots, r),
$$

whose coefficients $\xi_{\rho \sigma}(t)$ belong to $C^{\mu}\left(I_{1}, \mathbf{C}\right)$.

More exactly,

where

$$
\xi_{\rho \sigma}(t)=-\frac{B_{\rho \sigma}\left(\begin{array}{llll}
j_{1} & \jmath_{2} & \cdots & \jmath_{r} \\
k_{1} & k_{2} & \cdots & k_{r}
\end{array}\right)}{B\left(\begin{array}{llll}
\jmath_{1} & j_{2} & \cdots & \jmath_{r} \\
k_{1} & k_{2} & \cdots & k_{r}
\end{array}\right)} \quad\left(\begin{array}{l}
\rho=1,2, \cdots, r ; \\
\sigma=r+1, r+2, \cdots, n
\end{array}\right),
$$




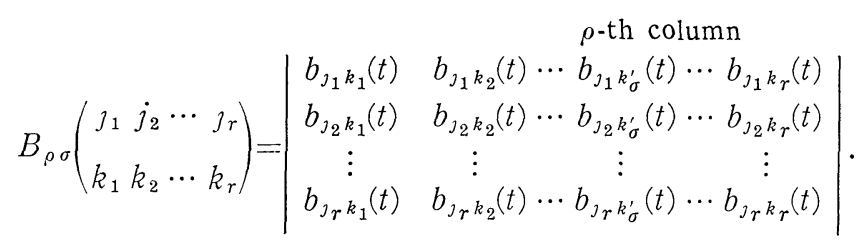

Of course, we see $\operatorname{rank} P(t)=s$ on $I_{1}$.

The matrix $Q(t)$ :

$$
Q(t)=\left(\begin{array}{cccc}
q_{11}(t) & q_{12}(t) & \cdots & q_{1 s}(t) \\
q_{21}(t) & q_{22}(t) & \cdots & q_{2 s}(t) \\
\vdots & \vdots & & \vdots \\
q_{n 1}(t) & q_{n 2}(t) & \cdots & q_{n s}(t)
\end{array}\right)
$$

also has a structure similar to $P(t)$.

We can choose arbitrarily all components $q_{m_{\sigma}^{\prime} g}(t)$ of $s$ row vectors

$$
\hat{\boldsymbol{q}}_{m_{\sigma}^{\prime}}(t)=\left(q_{m_{\sigma}^{\prime} 1}(t), q_{m_{\sigma}^{\prime} 2}(t), \cdots, q_{m_{\sigma}^{\prime} s}(t)\right) \quad(\sigma=r+1, r+2, \cdots, n)
$$

or $s$ column vectors

$$
\tilde{\boldsymbol{q}}_{g}(t)=\operatorname{col}\left(q_{m_{r+1}^{\prime}} g(t), q_{m_{r+2}^{\prime}} g(t), \cdots, q_{m_{n}^{\prime} g}(t)\right) \quad(g=1,2, \cdots, s)
$$

under restrictions that $q_{m_{\sigma}^{\prime} g}(t) \in C^{\mu}\left(I_{2}, \mathbf{C}\right)$ and

$$
\operatorname{det}\left(\tilde{\boldsymbol{q}}_{1}(t), \tilde{\boldsymbol{q}}_{2}(t), \cdots, \tilde{\boldsymbol{q}}_{s}(t)\right)=\operatorname{det}\left(\begin{array}{c}
\hat{\boldsymbol{q}}_{m_{r+1}^{\prime}}(t) \\
\hat{\boldsymbol{q}}_{m_{r+2}^{\prime}}(t) \\
\vdots \\
\hat{\boldsymbol{q}}_{m_{n}^{\prime}}(t)
\end{array}\right) \neq 0 \quad \text { on } \quad I_{2} .
$$

Other $r$ row vectors

$$
\hat{\boldsymbol{q}}_{m} \rho(t)=\left(q_{m_{\rho^{1}}}(t), q_{m} \rho^{2}(t), \cdots, q_{m} \rho^{s}(t)\right) \quad(\rho=1,2, \cdots, r)
$$

can be expressed by linear combinations of $\hat{\boldsymbol{q}}_{m_{\sigma}^{\prime}}(t)(\sigma=r+1, r+2, \cdots, n)$ :

$$
\hat{\boldsymbol{q}}_{m_{\rho}}(t)=\sum_{\sigma=r+1}^{n} \eta_{\rho \sigma}(t) \hat{\boldsymbol{q}}_{m_{\sigma}^{\prime}}(t) \quad(\rho=1,2, \cdots, r),
$$

whose coefficients $\eta_{\rho \sigma}(t)$ belong to $C^{\mu}\left(I_{2}, \mathbf{C}\right)$.

Of course, we have $\operatorname{rank} Q(t)=s$ on $I_{2}$.

3. We use the same notations as in Nos. 1-2, and will prove the following:

LEMMA 1. Assume that the condition (1) is satisfied on $I_{1} \cup I_{2}$ and the conditions (3) and (4) are satisfied on $I_{1}$ and on $I_{2}$ respectively. Then we have 
on $I_{1} \cap I_{2}$.

$$
\begin{aligned}
& B\left(\begin{array}{cccc}
l_{1} & l_{2} & \cdots & l_{r} \\
k_{1} & k_{2} & \cdots & k_{r}
\end{array}\right) \neq 0 ; \\
& B\left(\begin{array}{cccc}
j_{1} & j_{2} & \cdots & j_{r} \\
m_{1} & m_{2} & \cdots & m_{r}
\end{array}\right) \neq 0
\end{aligned}
$$

Proof. The lemma shall be principally proved for (9). If we put

$$
\hat{\boldsymbol{b}}_{l_{\rho}}(t)=\left(b_{l_{\rho} m_{1}}(t), b_{l_{\rho} m_{2}}(t), \cdots, b_{l_{\rho} m_{r}}(t)\right) \quad(\rho=1,2, \cdots, r),
$$

then, by virtue of the conditions (1) and (4), other vectors

$$
\hat{\boldsymbol{b}}_{l_{\sigma}^{\prime}}(t)=\left(b_{l_{\sigma}^{\prime} m_{1}}(t), b_{l_{\sigma}^{\prime} m_{2}}(t), \cdots, b_{l_{\sigma}^{\prime} m_{r}}(t)\right) \quad(\sigma=r+1, r+2, \cdots, n)
$$

can be expressed by linear combinations of $\hat{\boldsymbol{b}}_{l_{\rho}}(t)(\rho=1,2, \cdots, r)$ with coefficients $\varphi_{\rho \sigma}(t)$ belonging to $C^{\mu}\left(I_{2}, \mathbf{C}\right)$. That is,

$$
\hat{\boldsymbol{b}}_{l_{\sigma}^{\prime}}(t)=\sum_{\rho=1}^{r} \varphi_{\rho \sigma}(t) \hat{\boldsymbol{b}}_{l_{\rho}}(t) \quad(\sigma=r+1, r+2, \cdots, n),
$$

where

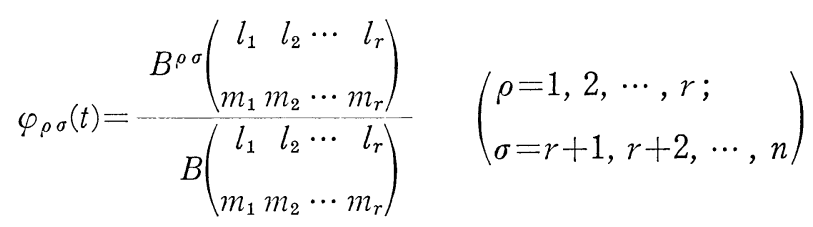

and

$$
B^{\rho \sigma}\left(\begin{array}{cccc}
l_{1} & l_{2} & \cdots & l_{r} \\
m_{1} & m_{2} & \cdots & m_{r}
\end{array}\right)=\left|\begin{array}{cccc}
b_{l_{1} m_{1}}(t) & b_{l_{1} m_{2}}(t) & \cdots & b_{l_{1} m_{r}}(t) \\
b_{l_{2} m_{1}}(t) & b_{l_{2} m_{2}}(t) & \cdots & b_{l_{2} m_{r}}(t) \\
\vdots & \vdots & & \vdots \\
b_{l_{\sigma}^{\prime} m_{1}}(t) & b_{l_{\sigma}^{\prime} m_{2}}(t) & \cdots & b_{l_{\sigma}^{\prime} m_{r}}(t) \\
\vdots & \vdots & & \vdots \\
b_{l_{r} m_{1}}(t) & b_{l_{r} m_{2}}(t) & \cdots & b_{l_{r} m_{r}}(t)
\end{array}\right| \rho \text {-th row }
$$

In this case, any component $b_{l_{\sigma}^{\prime} m_{\nu}^{\prime}}(t)(\sigma, v=r+1, r+2, \cdots, n)$ which does not lie on the $m_{\tau}$-th column $(\tau=1,2, \cdots, r)$ of $B(t)$ also can be expressed by a linear combination with the common coefficients $\varphi_{\rho \sigma}(t)$ as follows:

$$
b_{l_{\sigma}^{\prime} m_{\nu}^{\prime}}(t)=\sum_{\rho=1}^{r} \varphi_{\rho \sigma}(t) b_{l_{\rho} m_{\nu}^{\prime}}(t) \quad(\sigma, v=r+1, r+2, \cdots, n),
$$

otherwise it contradicts the fact that the condition (1) holds on $I_{2}$.

Next if we put

$$
\tilde{\boldsymbol{b}}_{k_{\tau}}(t)=\operatorname{col}\left(b_{\jmath_{1} k_{\tau}}(t), b_{\jmath_{2} k_{\tau}}(t), \cdots, b_{\jmath_{r} k_{\tau}}(t)\right) \quad(\tau=1,2, \cdots, r),
$$


then, by virtue of the conditions (1) and (3), other vectors

$$
\tilde{\boldsymbol{b}}_{k_{\nu}^{\prime}}(t)=\operatorname{col}\left(b_{\rho_{1} k_{\nu}^{\prime}}(t), b_{\partial_{2} k_{\nu}^{\prime}}(t), \cdots, b_{\jmath_{r} k_{\nu}^{\prime}}(t)\right) \quad(\nu=r+1, r+2, \cdots, n)
$$

can be expressed by linear combinations of $\widetilde{\boldsymbol{b}}_{k_{\tau}}(t)(\tau=1,2, \cdots, r)$ with coefficients belonging to $C^{\mu}\left(I_{1}, \mathbf{C}\right)$. That is,

$$
\widetilde{\boldsymbol{b}}_{k_{\nu}^{\prime}}(t)=\sum_{\tau=1}^{r} \phi_{\tau \nu}(t) \tilde{\boldsymbol{b}}_{k_{\tau}}(t) \quad(\nu=r+1, r+2, \cdots, n),
$$

where

$$
\phi_{\tau v}(t)=\frac{B_{\tau v}\left(\begin{array}{llll}
\jmath_{1} & \jmath_{2} & \cdots & \jmath_{r} \\
k_{1} & k_{2} & \cdots & k_{r}
\end{array}\right)}{B\left(\begin{array}{llll}
\jmath_{1} & \jmath_{2} & \cdots & \jmath_{r} \\
k_{1} & k_{2} & \cdots & k_{r}
\end{array}\right)} \quad\left(\begin{array}{l}
\tau=1,2, \cdots, r ; \\
v=r+1, r+2, \cdots, n
\end{array}\right)
$$

and

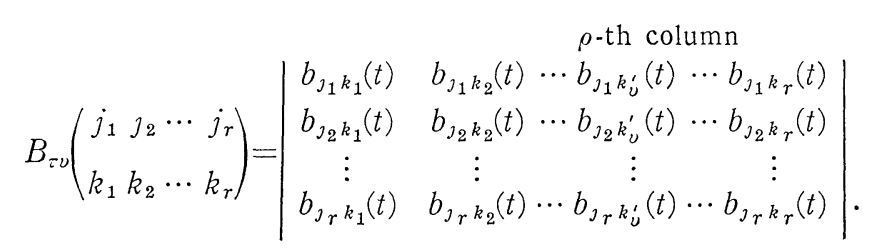

In this case, any component $b_{j_{\sigma}^{\prime}}^{\prime}(t)(\sigma, v=r+1, r+2, \cdots, n)$ which does not lie on the $j_{\rho}$-th row $(\rho=1,2, \cdots, r)$ of $B(t)$ also can be expressed by a linear combination with the common coefficients $\psi_{\tau v}(t)$ as follows:

$$
b_{j_{\sigma}^{\prime} k_{\nu}^{\prime}}(t)=\sum_{\tau=1}^{r} \psi_{\tau \nu}(t) b_{j_{\sigma}^{\prime} k_{\tau}}(t) \quad(\sigma, v=r+1, r+2, \cdots, n),
$$

otherwise it contradicts the fact that the condition (1) holds on $I_{1}$.

Under these circumstances, we arrive at the following conclusion.

Multiplying an adequate function which belongs to $C^{\mu}\left(I_{2}, \mathbf{C}\right)$, to each $l_{\rho}$-th row vector $(\rho=1,2, \cdots, r)$ of $B(t)$ and adding these row vectors to each $l_{\sigma}^{\prime}$-th row vector $(\sigma=r+1, r+2, \cdots, n)$ of $B(t)$, we can make each $l_{\sigma}^{\prime}$-th row vector be the zero vector. And further, multiplying an adequate function which belongs to $C^{\mu}\left(I_{1}, \mathbf{C}\right)$, to each $k_{\tau}$-th column vector $(\tau=1,2, \cdots, r)$ of $B(t)$ and adding these column vectors to each $k_{v}^{\prime}$-th column vector $(v=r+1, r+2, \cdots, n)$ of $B(t)$, we can make each $k_{v}^{\prime}$-th column vector be the zero vector. After all, there remain only the components $b_{l_{\rho} k_{\tau}}(t)(\rho, \tau=1,2, \cdots, r)$ in $B(t)$, and hence the condition (1) implies

$$
B\left(\begin{array}{cccc}
l_{1} & l_{2} & \cdots & l_{r} \\
k_{1} & k_{2} & \cdots & k_{r}
\end{array}\right) \neq 0 \quad \text { on } \quad I_{1} \cap I_{2} .
$$

We also can prove, on the same lines, that 


$$
B\left(\begin{array}{cccc}
j_{1} & \jmath_{2} & \cdots & \jmath_{r} \\
m_{1} & m_{2} & \cdots & m_{r}
\end{array}\right) \neq 0 \quad \text { on } \quad I_{1} \cap I_{2} \text {. }
$$

4. We use the same notations as in Nos. 1-2, and for the solutions $P(t)$ and $Q(t)$ obtained in No. 2, we will prove the following:

LeMma 2. Suppose that the condition (1) holds on $I_{1} \cup I_{2}$ and the condition (3) holds on $I_{1}$ and the condition (4) holds on $I_{2}$. Let $P(t)$ and $Q(t)$ be the solutions of the equations (5) and (6) respectively, obtained in No. 2. Then there exists a square matrix $C(t)$ of degree s such that

(I) Every component $c_{\jmath k}(t)$ of $C(t)$ belongs to $C^{\mu}\left(I_{1} \cap I_{2}, \mathbf{C}\right)$;

(II) $\operatorname{rank} C(t)=s$ on $I_{1} \cap I_{2}$;

(III) $P(t)=Q(t) C(t) \quad$ on $\quad I_{1} \cap I_{2}$.

Proof. By rearranging the row vectors of $P(t)$ and $Q(t)$, we put

$$
\hat{P}(t)=\left(\begin{array}{c}
\hat{\boldsymbol{p}}_{k_{1}}(t) \\
\vdots \\
\hat{\boldsymbol{p}}_{k_{r}}(t) \\
\hat{\boldsymbol{p}}_{k_{r+1}^{\prime}}(t) \\
\vdots \\
\hat{\boldsymbol{p}}_{k_{n}^{\prime}}^{\prime}(t)
\end{array}\right) \quad \text { and } \quad \hat{Q}(t)=\left(\begin{array}{c}
\hat{\boldsymbol{q}}_{k_{1}}(t) \\
\vdots \\
\hat{\boldsymbol{q}}_{k_{r}}(t) \\
\hat{\boldsymbol{q}}_{k_{r+1}^{\prime}}(t) \\
\vdots \\
\hat{\boldsymbol{q}}_{k_{n}^{\prime}}^{\prime}(t)
\end{array}\right) .
$$

Then, by virtue of the condition (9) proved in Lemma 1 , we can express the vectors $\hat{\boldsymbol{p}}_{k_{1}}(t), \hat{\boldsymbol{p}}_{k_{2}}(t), \cdots, \hat{\boldsymbol{p}}_{k_{r}}(t)$ by linear combinations of $\hat{\boldsymbol{p}}_{k_{r+1}^{\prime}}(t), \hat{\boldsymbol{p}}_{k_{r+2}^{\prime}}(t), \cdots$, $\hat{\boldsymbol{p}}_{k_{n}^{\prime}}(t)$ and the vectors $\hat{\boldsymbol{q}}_{k_{1}}(t), \hat{\boldsymbol{q}}_{k_{2}}(t), \cdots, \hat{\boldsymbol{q}}_{k_{r}}(t)$ by linear combinations of $\hat{\boldsymbol{q}}_{k_{r+1}^{\prime}}(t), \hat{\boldsymbol{q}}_{k_{r+2}^{\prime}}(t), \cdots, \hat{\boldsymbol{q}}_{k_{n}^{\prime}}(t)$ with the same coefficients belonging to $C^{\mu}\left(I_{1} \cap I_{2}, \mathbf{C}\right)$. That is,

$$
\begin{cases}\hat{\boldsymbol{p}}_{k_{\rho}}(t)=\sum_{\sigma=r+1}^{n} \zeta_{\rho \sigma}(t) \hat{\boldsymbol{p}}_{k_{\sigma}^{\prime}}(t) & (\rho=1,2, \cdots, r) ; \\ \hat{\boldsymbol{q}}_{k_{\rho}}(t)=\sum_{\sigma=r+1}^{n} \zeta_{\rho \sigma}(t) \hat{\boldsymbol{q}}_{k_{\sigma}^{\prime}}(t) & (\rho=1,2, \cdots, r),\end{cases}
$$

where $\zeta_{\rho \sigma}(t) \in C^{\mu}\left(I_{1} \cap I_{2}, \mathbf{C}\right)$ and more exactly

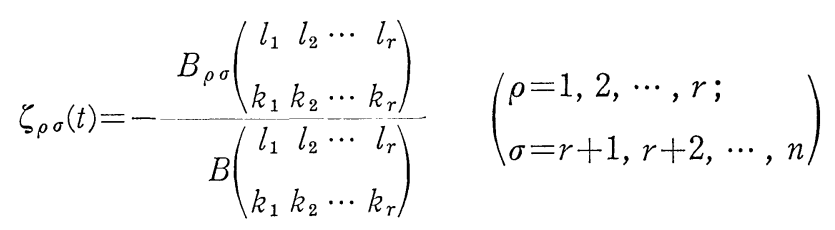

and 


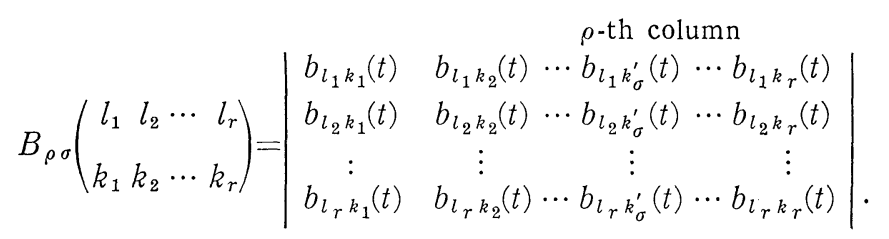

Now, we shall verify

$$
\operatorname{det}\left(\begin{array}{c}
\hat{\boldsymbol{q}}_{k_{r+1}^{\prime}}(t) \\
\hat{\boldsymbol{q}}_{k_{r+2}^{\prime}}(t) \\
\hat{\boldsymbol{q}}_{k_{n}^{\prime}}(t)
\end{array}\right) \neq 0 \quad \text { on } \quad I_{1} \cap I_{2} .
$$

If the above determinant vanishes at some $t_{0} \in I_{1} \cap I_{2}$, then there exists a set of $s$ complex numbers: $\left(c_{1}, c_{2}, \cdots, c_{s}\right) \neq(0,0, \cdots, 0)$ such that

$$
c_{1} \hat{\boldsymbol{q}}_{k_{r+1}^{\prime}}\left(t_{0}\right)+c_{2} \hat{\boldsymbol{q}}_{k_{r+2}^{\prime}}\left(t_{0}\right)+\cdots+c_{s} \hat{\boldsymbol{q}}_{k_{n}^{\prime}}\left(t_{0}\right)=\boldsymbol{o} .
$$

This fact and the fact that $\hat{\boldsymbol{q}}_{k_{\rho}}(t)(\rho=1,2, \cdots, r)$ can be expressed by linear combinations of $\hat{\boldsymbol{q}}_{k_{\sigma}^{\prime}}(t)(\sigma=r+1, r+2, \cdots, n)$, imply

$$
\operatorname{rank}\left(\begin{array}{c}
\hat{\boldsymbol{q}}_{k_{1}}\left(t_{0}\right) \\
\vdots \\
\hat{\boldsymbol{q}}_{k_{r}}\left(t_{0}\right) \\
\hat{\boldsymbol{q}}_{k_{r+1}^{\prime}}\left(t_{0}\right) \\
\vdots \\
\hat{\boldsymbol{q}}_{k_{n}^{\prime}}^{\prime}\left(t_{0}\right)
\end{array}\right)<s
$$

which contradicts that $\operatorname{rank} Q(t)=s$ on $I_{2}$.

Thus there exists a square matrix $C(t)$ of degree $s$ such that

$$
\left(\begin{array}{c}
\hat{\boldsymbol{p}}_{k_{r+1}^{\prime}}(t) \\
\hat{\boldsymbol{p}}_{k_{r+2}^{\prime}}(t) \\
\vdots \\
\hat{\boldsymbol{p}}_{k_{n}^{\prime}}(t)
\end{array}\right)=\left(\begin{array}{c}
\hat{\boldsymbol{q}}_{k_{r+1}^{\prime}}^{\prime}(t) \\
\hat{\boldsymbol{q}}_{k_{r+2}^{\prime}}^{\prime}(t) \\
\vdots \\
\hat{\boldsymbol{q}}_{k_{n}^{\prime}}(t)
\end{array}\right) C(t) \quad \text { on } \quad I_{1} \cap I_{2}
$$

and every component of $C(t)$ belongs to $C^{\mu}\left(I_{1} \cap I_{2}, \mathbf{C}\right)$. It follows furthermore from (7) that $\operatorname{det} C(t) \neq 0$ on $I_{1} \cap I_{2}$, and hence $\operatorname{rank} C(t)=s$ on $I_{1} \cap I_{2}$.

By rearranging the row vectors of $\hat{P}(t)$ and $\hat{Q}(t)$, and by observing the relations (11), we have

$$
P(t)=Q(t) C(t) \quad \text { on } \quad I_{1} \cap I_{2} .
$$

Thus, the lemma has been completely proved.

5. Now we will prove the following: 
THEOREM. Let $I$ be a closed interval $[\alpha, \beta]$ of a real variable $t$ and let $B(t)=\left(b_{j k}(t)\right)$ be a square matrix of degree $n$ whose components $b_{j k}(t)$ belong to $C^{\mu}(I, \mathbf{C})$ and further assume that the condition $(1)$ is satisfied on $I$. Then there exists an $n \times s$ matrix $P(t)=\left(p_{j k}(t)\right)$ such that $P(t)$ satisfies the equation (2) on $I$ and all components $p_{j k}(t)$ of $P(t)$ belong to $C^{\mu}(I, \mathbf{C})$ and $\operatorname{rank} P(t)=s$ on $I$.

Proof. Let us put $r=n-s$. Then, for any point $t_{0} \in[\alpha, \beta]$, there exists, by assumption, a nonzero minor of degree $r$ of $B\left(t_{0}\right)$. Furthermore, there exists, by virtue of the continuity of functions, a neighborhood $U\left(t_{0}\right)$ of $t_{0}$ such that the above-mentioned minor does not vanish on $I \cap U\left(t_{0}\right)$.

Since we can find such neighborhoods $U(t)$ for all points $t \in I$ and since we see $I \subset \bigcup_{t \in I} U(t)$, this open covering $\{U(t)\}_{t \in I}$ has, by the Heine-Borel theorem, a finite subcovering $\left\{U\left(t_{k}\right)\right\}_{k=1}^{\alpha_{0}}$. By use of this subcovering, we can form, without loss of generality, a set $\left\{I_{k}\right\}_{k=1}^{\kappa_{0}^{0}}$ of intervals possessing the following properties :

(i) $I=\sum_{\kappa=1}^{\kappa 0} I_{\kappa}$;

(ii) $I_{1}=\left[\alpha_{1}, \beta_{1}\right), \quad I_{\kappa_{0}}=\left(\alpha_{\kappa_{0}}, \beta_{\kappa_{0}}\right], \quad \alpha_{1}=\alpha, \quad \beta_{\kappa_{0}}=\beta$,

$I_{\kappa}=\left(\alpha_{\kappa}, \beta_{\kappa}\right) \quad\left(\kappa=2,3, \cdots, \kappa_{0}-1\right)$;

(iii) $I_{\kappa} \cap I_{\kappa+1} \neq \varnothing \quad\left(\kappa=1,2, \cdots, \kappa_{0}-1\right)$,

$I_{\kappa} \cap I_{\kappa^{\prime}}=\varnothing \quad\left(\kappa+1<\kappa^{\prime}, \kappa=1,2, \cdots, \kappa_{0}-2\right)$,

that is, $\alpha_{1}<\alpha_{2}<\beta_{1}<\cdots<\alpha_{\kappa}<\beta_{k-1}<\alpha_{\kappa+1}<\beta_{\kappa}$

$<\cdots<\beta_{\kappa_{0}-2}<\alpha_{\kappa_{0}}<\beta_{\kappa_{0}-1}<\beta_{\kappa_{0}} \quad\left(\kappa=2,3, \cdots, \kappa_{0}-1\right) ;$

(iv) For each $I_{k}$, there exists a minor of degree $r$ of $B(t)$ which does not vanish on $I_{n}$.

We consider first the intervals $I_{1}$ and $I_{2}$, and we choose two minors $B\left(\begin{array}{llll}\jmath_{1} & \jmath_{2} & \cdots & \jmath_{r} \\ k_{1} & k_{2} & \cdots & k_{r}\end{array}\right)$ and $B\left(\begin{array}{cccc}l_{1} & l_{2} & \cdots & l_{r} \\ m_{1} & m_{2} & \cdots & m_{r}\end{array}\right)$ of degree $r$ of $B(t)$ such that

$$
B\left(\begin{array}{cccc}
\jmath_{1} & \jmath_{2} & \cdots & \jmath_{r} \\
k_{1} & k_{2} & \cdots & k_{r}
\end{array}\right) \neq 0 \text { on } I_{1} \text { and } B\left(\begin{array}{cccc}
l_{1} & l_{2} & \cdots & l_{r} \\
m_{1} & m_{2} & \cdots & m_{r}
\end{array}\right) \neq 0 \text { on } I_{2} \text {. }
$$

As seen in No. 2, there exist, in this case, the matrices $P(t)$ and $Q(t)$ satisfying the equations (5) and (6) respectively, such that all components $p_{j k}(t)$ of $P(t)$ belong to $C^{\mu}\left(I_{1}, \mathbf{C}\right)$ and all components $q_{j k}(t)$ of $Q(t)$ belong to $C^{\mu}\left(I_{2}, \mathbf{C}\right)$ and further

$$
\operatorname{rank} P(t)=s \quad \text { on } I_{1} \quad \text { and } \operatorname{rank} Q(t)=s \text { on } I_{2} \text {. }
$$

Now it follows from Lemma 2 that there exists a square matrix $C(t)$ of degree $s$ possessing the properties (I), (II), (III) stated in Lemma 2.

We next use a method adopted in the paper of Y. Sibuya [1]. Since the matrix $C(t)$ is non-singular on $I_{1} \cap I_{2}$, if we choose an arbitrary point $t_{1} \in I_{1} \cap I_{2}$, 
and if we choose a sufficiently small positive number $\varepsilon$, then any square matrix $C$ of degree $s$ satisfying $\left\|C-C\left(t_{1}\right)\right\|<\varepsilon$, is non-singular, where $\|\cdot\|$ denotes the Euclidean norm of a matrix.

There exists, by virtue of the continuity of functions, a positive number $\delta$ such that $\left\|C(t)-C\left(t_{1}\right)\right\|<\varepsilon$ whenever $\left|t-t_{1}\right|<\delta$ and $t \in I_{1} \cap I_{2}$.

Let $t_{1}^{\prime}$ be a point belonging to $I_{1} \cap I_{2}$ such that $0<t_{1}^{\prime}-t_{1}<\delta$ and let $\gamma$ be a small positive number fulfilling the inequality $t_{1}+\gamma<t_{1}^{\prime}-\gamma$. Further let $\chi(t)$ be a real-valued function defined and of class $C^{\infty}$ on $-\infty<t<+\infty$, such that $0 \leqq \chi(t) \leqq 1$ for all $t, \chi(t)=1$ for $t \leqq t_{1}+\gamma$ and $\chi(t)=0$ for $t \geqq t_{1}^{\prime}-\gamma$.

And we make a square matrix $\widetilde{C}(t)$ of degree $s$ in the following manner:

Since

$$
\widetilde{C}(t)= \begin{cases}C(t) & \text { for } \alpha_{2}<t \leqq t_{1}, \\ \chi(t)\left(C(t)-C\left(t_{1}^{\prime}\right)\right)+C\left(t_{1}^{\prime}\right) & \text { for } t_{1} \leqq t \leqq t_{1}^{\prime}, \\ C\left(t_{1}^{\prime}\right) & \text { for } t_{1}^{\prime} \leqq t<+\infty .\end{cases}
$$

$$
\begin{aligned}
\| \widetilde{C}(t)- & C\left(t_{1}\right) \| \\
& =\left\|\chi(t)\left(C(t)-C\left(t_{1}\right)\right)+(1-\chi(t))\left(C\left(t_{1}^{\prime}\right)-C\left(t_{1}\right)\right)\right\| \\
& \leqq \chi(t)\left\|C(t)-C\left(t_{1}^{\prime}\right)\right\|+(1-\chi(t))\left\|C\left(t_{1}^{\prime}\right)-C\left(t_{1}\right)\right\| \\
& <\chi(t) \varepsilon+(1-\chi(t)) \varepsilon=\varepsilon
\end{aligned}
$$

for $t_{1} \leqq t \leqq t_{1}^{\prime}$, we see that $\tilde{C}(t)$ is non-singular on $\alpha_{2}<t<+\infty$.

Further we can easily verify that all components of $\widetilde{C}(t)$ are of class $\mathrm{C}^{\mu}$ on $\alpha_{2}<t<+\infty$.

We now put $P^{(1)}(t)=P(t)$ on $I_{1}$ and we define $P^{(2)}(t)$ on $I_{1} \cup I_{2}$ as follows:

$$
P^{(2)}(t)=\left\{\begin{array}{lll}
P^{(1)}(t) & \text { for } & \alpha_{1} \leqq t \leqq \alpha_{2}, \\
Q(t) \tilde{C}(t) & \text { for } & \alpha_{2}<t<\beta_{2} .
\end{array}\right.
$$

Then $P^{(2)}(t)$ is a solution of the equation (2) on $I_{1} \cup I_{2}$ and all components of $P^{(2)}(t)$ belong to $C^{\mu}\left(I_{1} \cup I_{2}, \mathbf{C}\right)$ and further

$$
\operatorname{rank} P^{(2)}(t)=s \quad \text { on } I_{1} \cup I_{2} \text {. }
$$

By repeating the above-mentioned process for the intervals $I_{k}(\kappa=1,2, \cdots$, $\kappa_{0}$ ) successively, we can construct the desired solution $P(t)$ of the equation (2) on $I$.

\section{BIBLIOGRAPHY}

[1] Y. Sibuya, Some global properties of matrices of functions of one variable, Math. Annalen 161 (1965), 66-77.

Department of Mathematics

Tokyo Institute of TechNology 